\title{
Automatic Freezing-Tolerant Rapeseed Material Recognition Using UAV Images and Deep Learning
}

\section{Lili Li}

Wuhan University https://orcid.org/0000-0003-4972-1848

\section{Jiangwei Qiao}

Oil Crops Research Institute of the Chinese Academy of Agricultural Sciences

Jian Yao

Wuhan University

Jie Li

Hubei University of Technology

Li Li ( $\sim$ li.li@whu.edu.cn )

Wuhan University https://orcid.org/0000-0003-3138-6033

\section{Research}

Keywords: Freezing injury recognition, rapeseed, UAV images, deep learning, machine learning

Posted Date: October 14th, 2021

DOI: https://doi.org/10.21203/rs.3.rs-929591/v1

License: (c) (1) This work is licensed under a Creative Commons Attribution 4.0 International License. Read Full License

Version of Record: A version of this preprint was published at Plant Methods on January 13th, 2022. See the published version at https://doi.org/10.1186/s13007-022-00838-6. 


\title{
Automatic freezing-tolerant rapeseed material recognition using UAV images and deep learning
}

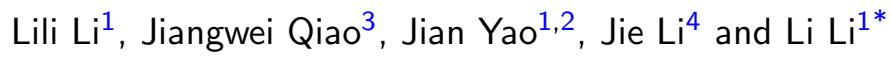

\footnotetext{
${ }^{*}$ Correspondence: li.li@whu.edu.cn

${ }^{1}$ School of Remote Sensing and

Information Engineering, Wuhan

University, Wuhan, China

Full list of author information is

available at the end of the article
}

\begin{abstract}
Background: Freezing injury is a serious and common damage that occurs to winter rapeseed during the overwintering period. The freezing injury directly reduces the rapeseed yield and causes serious economic loss. Thus, it is an important and urgent task for crop breeders to find the freezing-tolerant rapeseed materials in the process of breeding. Existing large-scale freezing-tolerant rapeseed material recognition methods mainly rely on the field investigation conducted by the agricultural experts using some professional equipment. These methods are time-consuming, inefficient and laborious. In addition, the accuracy of these traditional methods depends heavily on the knowledge and experience of experts.
\end{abstract}

Methods: To solve these problems of existing methods, we propose a low-cost freezing-tolerant rapeseed material recognition approach using deep learning technology and unmanned aerial vehicle (UAV) images captured by a consumer drone. We formulate the problem of freezing-tolerant material recognition as a binary classification problem, which can be solved well using deep learning technology. The proposed method can automatically and efficiently recognize the freezing-tolerant rapeseed materials from a large number of candidates. To train the deep learning network, we first manually construct the real dataset using the UAV images of rapeseed materials collected by the Phantom 4 Pro. Then, five classic deep learning networks (AlexNet, VGGNet16, ResNet18, ResNet50 and GoogLeNet) are selected to perform the freezing-tolerant rapeseed material recognition.

Result and Conclusion: The accuracy of the five deep learning networks used in our work is all over $92 \%$. Especially, ResNet50 provides the best accuracy $(93.33 \%)$ in this task. In addition, we also compare deep learning networks with traditional machine learning methods. The comparison results show that the deep learning-based approach significantly outperforms the traditional machine learning-based methods in our task. The experimental results show that it is feasible to recognize the freezing-tolerant rapeseed using UAV images and deep learning.

Keywords: Freezing injury recognition; rapeseed; UAV images; deep learning; machine learning

\section{Background}

As one of the most important economic crops in the world, rapeseed plays an important role in ensuring the supply of edible vegetable oil and nutrient-rich feed. Rapeseed oil is also an important source of biofuel. With the development of the global economy and the continuous improvement in the standard of living, the consumption demand of rapeseed drastically increases. However, because the arable 
land of rapeseed is usually limited, the supply of rapeseed in some countries is inadequate. For example, China is the largest edible oil consumption country in the world. However, due to the serious shortage of arable land, China is also the largest rapeseed oil importer country. In addition, as the price of fertilizer, pesticide and seed increases, the economic benefit of planting winter rapeseed decreases especially when the yield of rapeseed is not satisfactory. Thus, farmers are reluctant to plant rapeseed in many countries. In order to meet the increasing demand for rapeseed, it is imperative to expand the planting area and increase the per unit area output of rapeseed as much as possible.

There are many factors that influence the yield of rapeseed. Freezing injury is one of the most important factors that affect the growth of rapeseed. Winter rapeseed often suffers from serious freezing injury during the overwintering period. Low temperature will directly inhibit the metabolic response and prevent the expression of the full genetic potential of the plant [1]. The nutrient transport of rapeseed stem is affected by freezing injury, which leads to the seed setting rate and yield decreases. Thus, it is important for breeders to breed freezing-tolerant rapeseed materials. By selecting and breeding the freezing-tolerant rapeseed materials, it can expand the rapeseed planting area in cold regions. Rapeseed is better adapted to cold climates which can reduce the yield loss for freezing injury. However, in the past few years, the freezing-tolerant rapeseed material recognition still relies on field investigation conducted by agricultural experts. It is laborious, error-prone and inefficient for experts to select the freezing-tolerant materials from a large number of candidates. It is necessary to propose a new approach to recognize the freezing-tolerant materials automatically and efficiently. The development of remote sensing technology makes it possible.

In the field of remote sensing, the satellite image is one of the most powerful and important source data. Because the spectral characteristics of crops can be reflected in the satellite image, spectral information of the satellite image can express the relationship between natural environmental conditions and vegetation disaster. Satellite image can efficiently and effectively acquires the stress information of crops over a large-scale area. In recent years, satellite image has been widely applied to recognize the crop damage caused by drought $[2,3]$, flood $[4,5]$ and hail [6, 7], etc. Recently, many researchers also proposed to recognize the crop freezing injury using satellite image $[8,9]$. Because the normalized difference vegetation index (NDVI) of crop changes dramatically after suffering freezing injury. The NDVI is a prominent feature to analyze the freezing injury. Some studies compared and analyzed the NDVI calculated by satellite image before and after freezing injury to recognize the crop freezing damage [10-13]. However, the plant area of each candidate material is usually small (about 2 square meters). The satellite data with a limited spatial resolution can not recognize each material accurately. Thus, it is difficult to use satellite image to select the freezing-tolerant materials. With the development of UAVs, it provides a new source image to recognize the freezing-tolerant materials.

Compared with satellite image, the images captured by the sensors mounted on UAVs have a higher spatial resolution. Multispectral or hyperspectral images captured by the UAVs are one of the widely used source images in the field of agriculture. It can provide abundant and accurate spectral information of crops. Recently, 
many researchers proposed to use multispectral or hyperspectral images collected by UAVs to recognize the crop freezing injury [14-16]. In general, they firstly calculated different vegetation indices (VIs) using source images, and then several VIs are combined to recognize the freezing-injured crops. Je lowicki et al. [17] calculated six VIs (NDVI, NDVI_RE, OSAVI, OSAVI_RE, SAVI, SAVI_RE) from four spectral bands of multispectral UAV images. Then, the crop areas injured by freezing are recognized using the six VIs. Marin et al. [18] recognized and evaluated freezing damage of coffee plants in different zones using NDVI and Modified Simple Ratio (MSR) calculated from multispectral image. The result demonstrates that the NDVI and MSR have a strong relationship with the freezing injury. Their results demonstrate that using various VIs can effectively recognize the freezing injury of crops. However, because the acquisition and processing of multispectral or hyperspectral data are relatively complicated, it is hard for non-professional users to acquire high-quality multispectral images using the sensor mounted on an UAV platform. Nowadays, consumer UAVs equipped with an RGB camera provide a new choice to recognize freezing-tolerant materials. Compared with the professional UAV equipped with a multispectral sensor, the consumer UAV is cheap, convenient and flexible.

Nowadays, consumer UAVs equipped with an RGB camera have been widely used to monitor the crop growth process. With the continuous development of artificial intelligence (AI), the combination of UAVs and AI plays an important role in the development of precision agriculture. It has been widely applied to different tasks of agriculture, such as disease and insect detection [19, 20], yield prediction [21, 22], and crop lodging detection [23, 24]. Recently, the combination of RGB images collected by UAVs and AI techniques also have been applied to detect and classify the crop stresses [25-27]. Su et al. [28] developed a supervised learning system based on Support Vector Machine (SVM) to recognize the crop water stress using RGB images collected by an UAV. Firouz et al. [29] applied SVM to recognize the healthy and freezing damaged citrus fruits. However, the performance of traditional machine learning algorithms depends heavily on the hand-crafted features designed using the image information and prior knowledge. It is difficult to manually design the optimal features for different crop stress tasks. The traditional machine learning method is difficult to meet the requirement for crop stress recognition. With the rise of deep learning technologies, it proposes an intelligent solution for different crop stress tasks.

Nowadays, deep learning technology has made great breakthroughs and has been widely used in agricultural applications. The advantage of deep learning is that it provides powerful feature representations through end-to-end learning, the feature of images will be learned automatically by deep learning models [30]. In recent years, some researchers proposed to recognize the crop stresses using deep learning instead of traditional machine learning. Through the comparative experiments, they found that the accuracy of the crop stress recognition using deep learning is higher than using traditional machine learning algorithms [31, 32].

Compared to traditional machine learning, deep learning has better potential for the crop stress recognition. Yang et al. Yang et al. [33] adopted a convolutional neural network $(\mathrm{CNN})$ model to extract spectral features in the visible-near-infrared 
range. Then, they used the extracted spectral features to estimate freezing damage of corn seedlings. The freezing damage detected by CNN has a high correlation with the ranking given by chemical methods. It proved that spectral analysis based on the CNN model is suitable for recognizing the freezing damage in corn seedlings. Anami et al. [34] adopted a deep convolutional neural network (DCNN) framework to automatically recognize and classify various biotic and abiotic stress of rice using the RGB images collected by an UAV. The accuracy of the recognition result is $92.89 \%$, which proves the potential of deep learning in the recognition of crop stress. The fact proves that deep learning is more suitable for recognizing crop stress than traditional machine learning. It provides an effective way to assist experts to select stress-tolerant materials.

In this paper, we proposed a low-cost freezing injury recognition method using UAV images and deep learning technology. The main purpose of this research is to provide an approach that combines RGB images collected by a consumer UAV with deep learning to automatically recognize the freezing-tolerant rapeseed materials from a large number of breeding candidates. Then, the breeders can quickly assess and select the breeding materials from the freezing-tolerant materials that are preliminarily selected by deep learning. This approach can assist experts in selecting freezing-tolerant rapeseed materials quickly and precisely. It is an important step for breeders to conduct preliminary selecting from a large number of candidates. To the best of our knowledge, although many deep learning-based approaches are proposed to recognize crop stress, it is the first work to recognize the freezing-tolerant rapeseed material using deep learning and UAV images.

\section{Dataset and Methodology}

To automatically and efficiently recognize the freezing-tolerant rapeseed materials from a large number of candidates, we propose a new deep learning-based freezing injury recognition approach using the RGB images collected by a consumer UAV. The whole workflow of our proposed approach is presented in Fig. 1.

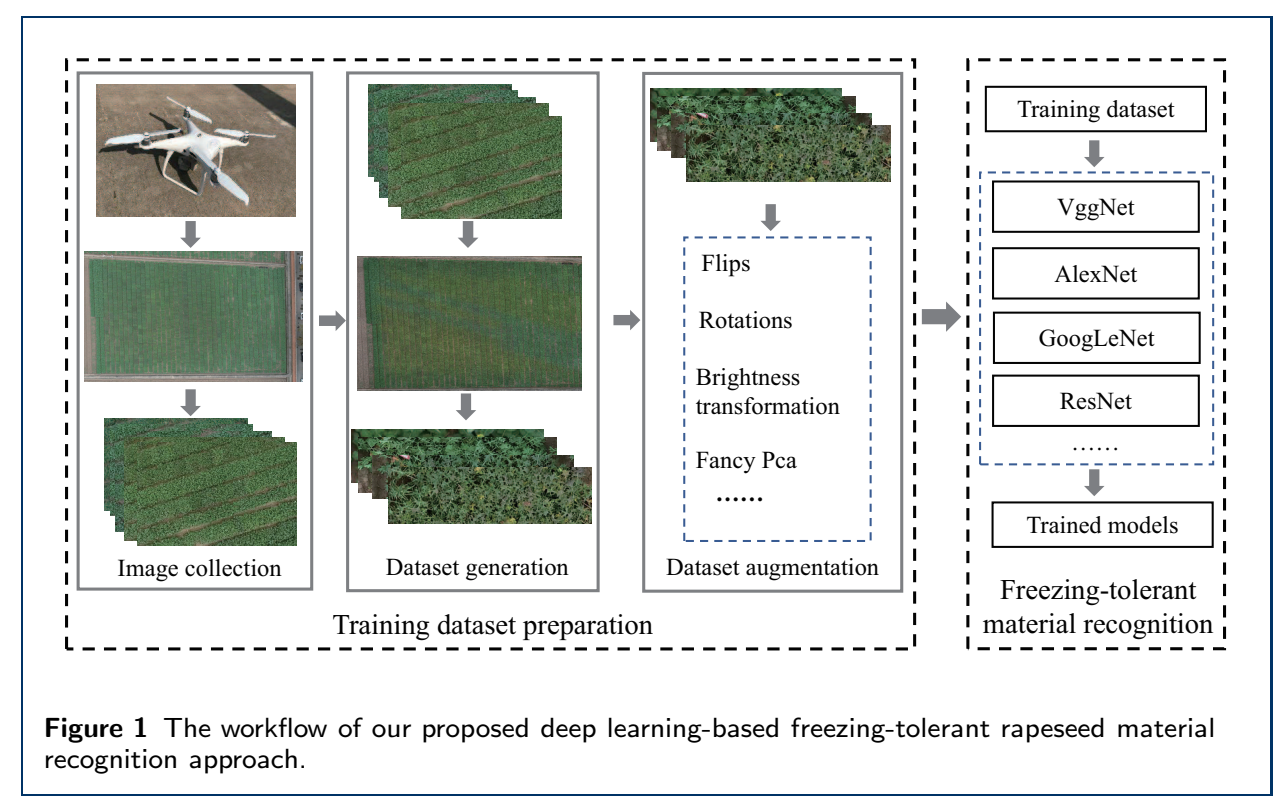




\section{Overview of study area and data source}

\section{Study area}

The experiment is conducted at the Oil Crops Research Institute, Chinese Academy of Agricultural Sciences, Wuhan, China (114 $31^{\prime} \mathrm{N}, 30^{\circ} 55^{\prime} \mathrm{E}$, and elevation $\left.20 \mathrm{~m}\right)$. The test site location is shown in Fig. 2. The test site is divided into many plots, the red box in Fig. 2 represents a plot. The width of each plot is $2 \mathrm{~m}$. Each plot consists of many rows. The line spacing of two adjacent rows is $16.7 \mathrm{~cm}$. Each rapeseed material consists of three rows. In the test site, there are many pure materials covering an area of about 0.45 ha. The rapeseed is sown on September 27th, 2020. To ensure that the growth of all materials before the overwintering period is normal, we carry out a continuous observation on rapeseed and take remedial measures for abnormal materials. The key goal of our proposed approach is to automatically and effectively select these freezing-tolerant materials from all materials using UAV images and deep learning technology.

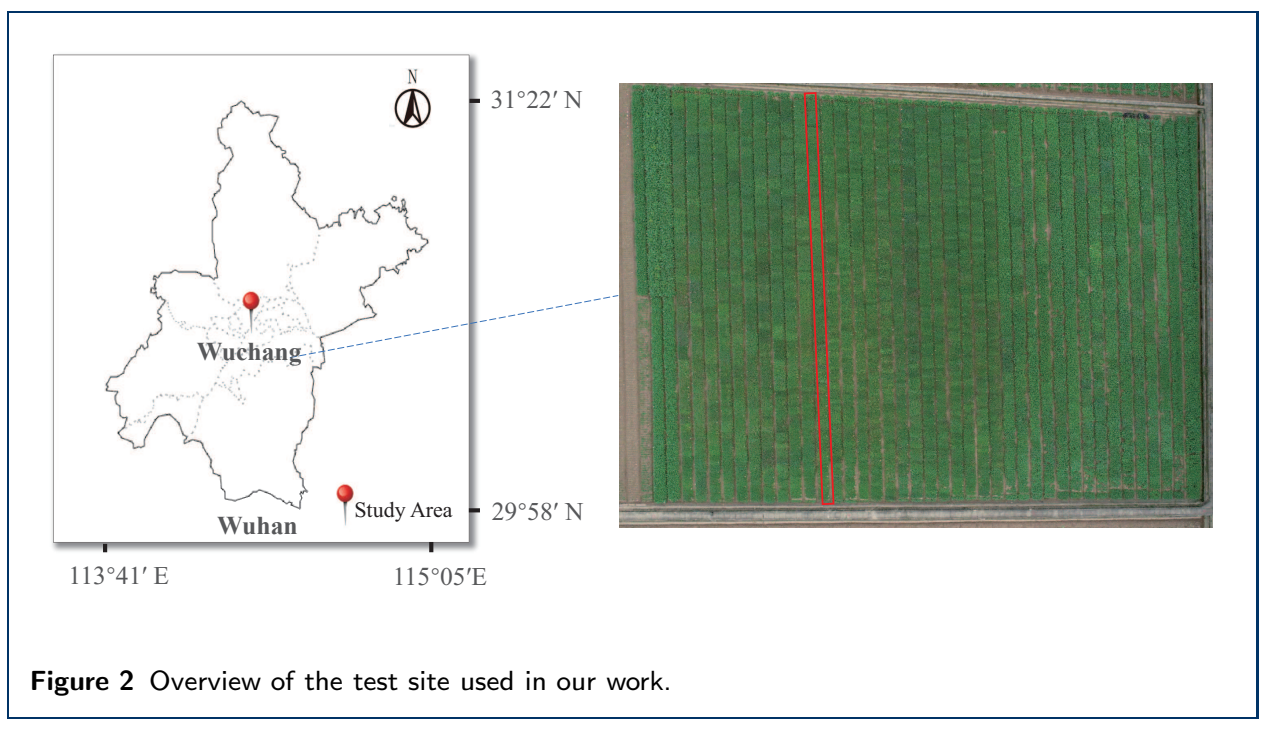

\section{Image acquisition}

In Fig. 3, we present the part of the historical temperature chart of Wuhan in November and December 2020. From the observation of the temperature chart and field investigation, we find that the rapeseed materials have suffered from the damage of low temperature around December 20th, 2020. In our experiments, we collect the UAV images of this test site on December 23th, 2020. The images are acquired by a DJI phantom4 Pro v2.0 (DJI, Shenzhen, China) equipped with an RGB camera with a spatial resolution of $5472 \times 3648$ pixels. The flight campaign is conducted from 3:00 pm to 3:30 pm. The weather is cloudy without wind. The flight height and speed are $10 \mathrm{~m}$ and $1.8 \mathrm{~m} / \mathrm{s}$, respectively. In order to generate the orthophoto map successfully, we set the frontal overlap and side overlap as $75 \%$ and $75 \%$, respectively.

\section{Dataset generation}

In this paper, we formulate the freezing-tolerant material recognition problem as a classification problem, which can be solved well using deep learning technology. 


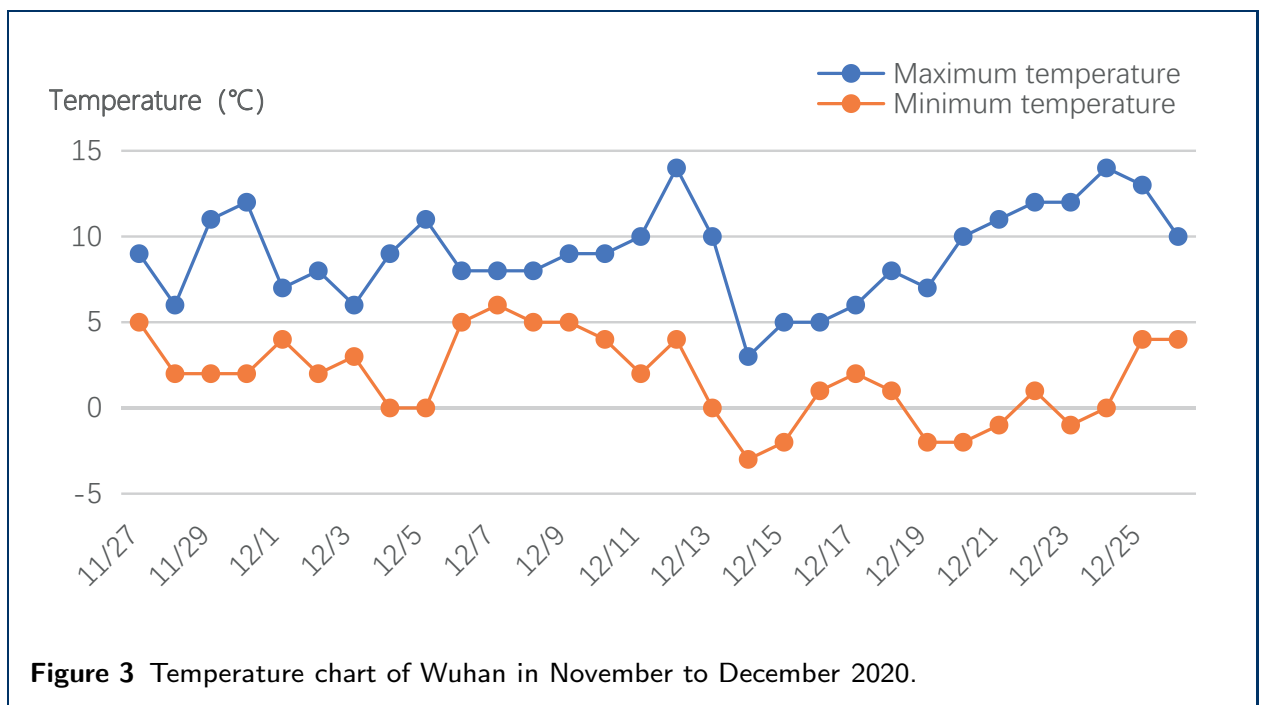

However, deep learning is a data-driven method, we need to prepare a large number of samples with ground truth to train the model. In this study, we directly apply the Agisoft PhotoScan software (Agisoft LLC, St. Petersburg, Russia) to generate the orthophoto map of the test site. Agisoft PhotoScan is excellent software, which is applied to automatically generate high-resolution real orthophoto map and DEM model. The orthophoto image is then used to crop and create the rapeseed freezing injury recognition dataset.

After the orthophoto map generation, the Adobe Photoshop software is used to cut out images to generate the experimental dataset. In the practical application of large-scale rapeseed fields, we can perform the batch cropping of images based on the geographic coordinates of the rapeseed fields, it can generate large amounts of cropped images automatically and efficiently. As mentioned in section of Study area, every material consists of three rows. Thus, we cut out the orthophoto map into 2847 samples, each sample represents a material that consists of three rows. All samples are resized to $600 \times 150$ after cropping. We invite the experts in the rapeseed breeding field to label the samples. The experts assign different labels to the freezinginjured and freezing-tolerant samples according to their experience and knowledge. The labels of the freezing-injured sample and freezing-tolerant sample are 1 and 0 , respectively. Then, we divide the whole dataset into training dataset and test dataset with an 8:2 ratio randomly. As a result, 2277 images are used to train the deep learning network, 570 images are used to test the accuracy of the trained model. The detailed number of samples in the training and test datasets is shown in Table 1.

Table 1 The number of freezing-injured and freezing-tolerant samples in the training and test datasets.

\begin{tabular}{ccc}
\hline Dataset & Freezing-tolerant & Freezing-injured \\
\hline Training dataset(80\%) & 572 & 1705 \\
Test dataset $(20 \%)$ & 94 & 476 \\
\hline
\end{tabular}

In Fig. 4, we present several representative freezing-injured and freezing-tolerant samples. From Fig. 4, we find that the symptoms of freezing-injured and freezingtolerant materials are different. Fiebelkorn and Rahman [1] pointed out that the 
symptoms of freezing-injured rapeseed can be seen on leaves, including leaf wilting, leaf bleaching, or in extreme cases, plant death. The obvious rapeseed freezing injury symptoms are shown in Fig. 4. The freezing-injured material has the following symptoms: the leaves turn yellow (Fig. 4(a)), the leave color is dark or fuchsia (Fig. 4(b)), the leave is overturned and the petiole has wet blotch (Fig. 4(c)). The leaves' color and petioles of the freezing-tolerant material are normal, as shown in Fig. 4(d)-(f).

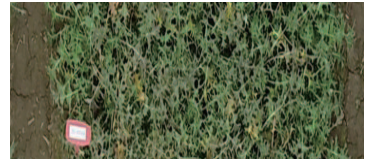

(a)

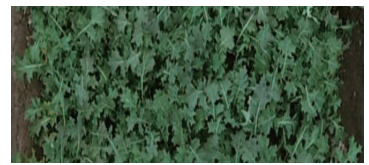

(d)

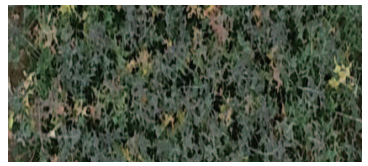

(b)

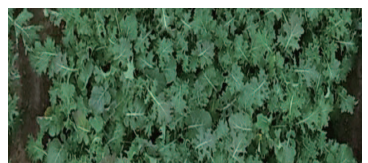

(e)

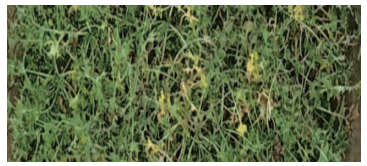

(c)

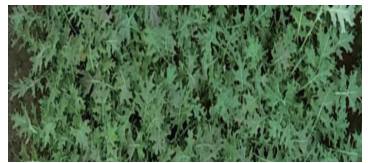

(f)

Figure 4 The symptoms of freezing-injured and freezing-tolerant materials. Freezing-injured material with yellowish leaves (a), dark, fuchsia leaves (b), overturned leaves and water-soaked petioles (c), (d)-(f) are freezing-tolerant materials with normal leaves' color and petioles.

\section{Dataset augmentation}

Deep learning is a data-driven approach, the number and quality of the training dataset will directly influence the effect of the trained model. In general, as the number of high-quality samples increases, the performance of the deep learning-based approach increases. However, in real applications, the number of high-quality samples is often insufficient. To get a high-quality trained model and improve the generalization ability of the model, we need to increase the number of the dataset. Data augmentation is a method that can augment the number and quality of training datasets. Data augmentation methods can be divided into supervised data augmentation and unsupervised data augmentation methods. We adopt supervised data augmentation methods in our work. Geometric transformation, brightness transformation, image stitching and fancy PCA (Principal Components Analysis) are used in our study. The results of these strategies are shown in Fig. 5. Next, we will give a brief introduction to each strategy.

To make the model more robust to the brightness changes, we use the brightness transformation, which is a common data augmentation strategy to augment the training data. We first transform the images from RGB color space to HSV color space. Then, we adjust the brightness of the original image. The original image brightness is adjusted to $80 \%, 90 \%, 110 \%$ and $120 \%$, respectively. The result of brightness transformation is shown in Fig. 5(b).

The geometric transformation is also a common image augmentation strategy, including rotation, flip operation, random rotation, etc. The rotation operation and flip operation are used to augment the dataset in this study. The results of geometric transformation are shown in Fig. 5(c) and Fig. 5(d)-(e), respectively. 


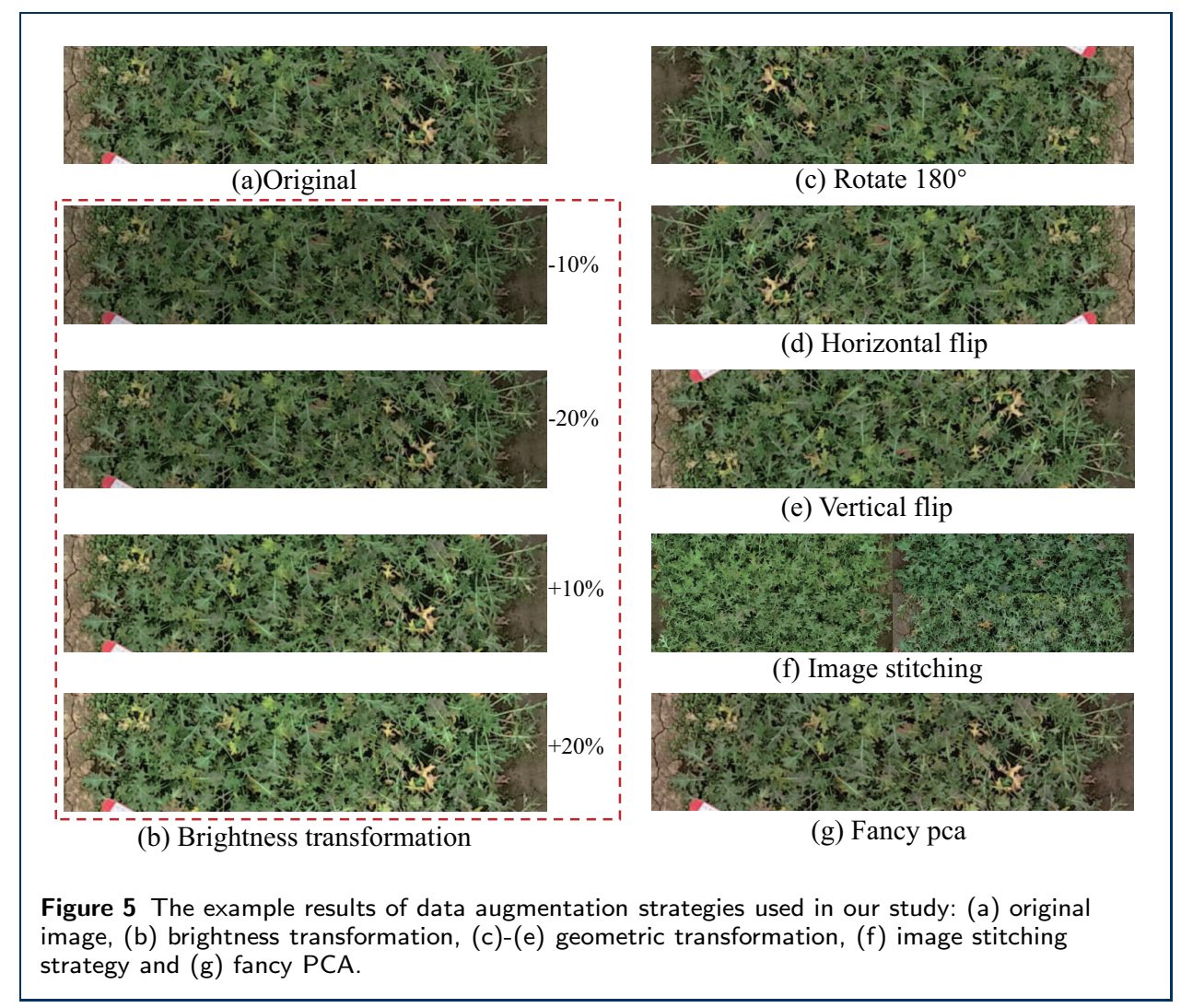

As shown in Fig. 4, there are many symptoms of freezing-injured rapeseed. In the real environment, the situation of freezing injury is more complex. In order to improve the generalization ability of the model for the case of freezing injury, we adopt the diagonal stitching strategy. We randomly select four images with the same label in the dataset and stitch the four images into one new image. Then, we resize the stitching image to the original image size of $600 \times 150$. The result of the diagonal stitching strategy is shown in Fig. 5(f).

Fancy PCA as a common data augmentation strategy is firstly proposed in the study of AlexNet [35]. Fancy PCA changes the intensities of the RGB channel along with the natural variation of the images and performs PCA on the color channels. Firstly, PCA is performed on all RGB pixel values to obtain the eigenvectors $(\mathrm{P} 1, \mathrm{P} 2, \mathrm{P} 3)$ and eigenvalues $(\lambda 1, \lambda 2, \lambda 3)$, and then a set of random values $(\alpha 1, \alpha 2, \alpha 3)$ from a Gaussian distribution with mean=0 and standard deviation $=0.1$ times eigenvalues to get the $[\alpha 1 \lambda 1, \alpha 2 \lambda 2, \alpha 3 \lambda 3]$. Finally, to each RGB image pixel $I x y=\left[I x y^{R}, I x y^{G}, I x y^{B}\right]$, the quantity $[P 1, P 2, P 3][\alpha 1 \lambda 1, \alpha 2 \lambda 2, \alpha 3 \lambda 3]^{T}$ is added. The description detail of the Fancy PCA is reported in [35]. Fancy PCA is used to augment the dataset in this study, the result of fancy PCA is shown in Fig. 5(g).

\section{Architectures of CNNs}

To effectively distinguish between freezing-injured and freezing-tolerant materials, we propose to apply a convolutional neural network (CNN) to classify the images of all materials. CNN is a classical neural network that has been widely applied to 
analyze the visual image. In recent years, CNN has been applied to analyze crop images. In Fig. 6, we present a basic CNN architecture designed for the classification of rapeseed materials. In general, a CNN architecture consists of a set of convolutional, pooling and fully connected layers. In our paper, we directly select several classical CNN architectures to complete the task for the freezing-tolerant rapeseed material recognition. Next, we will briefly introduce the selected networks.

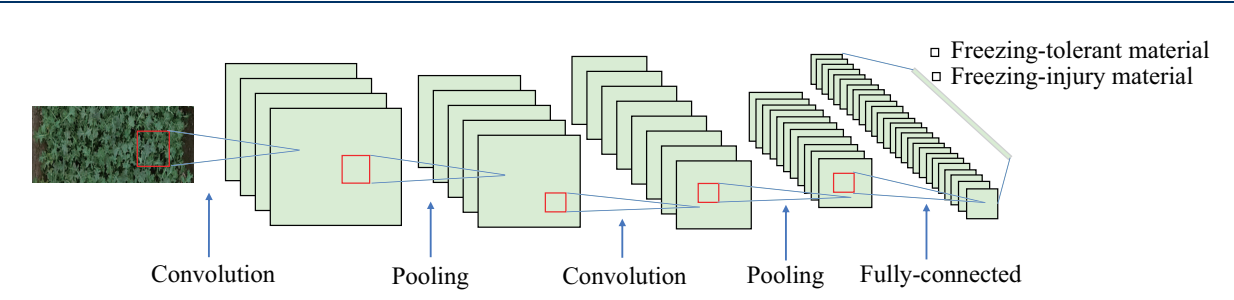

Figure 6 Architecture of the classical CNN.

AlexNet

AlexNet [35] is one of the most popular CNN architectures, and it is also the first $\mathrm{CNN}$ to win the ImageNet contest. In addition, AlexNet is the first network to implement a deep convolutional neural network structure on a large-scale image dataset. AlexNet architecture consists of 5 convolutional layers, 3 max-pooling layers, 2 normalization layers, 2 fully connected layers, and 1 softmax layer. In AlexNet architecture, it uses the Rectified Linear Unit (ReLU) activation function instead of the sigmoid or tanh activation function. Krizhevsky et al.[35] found out that using ReLU as an activation function can significantly accelerate the speed of the training process. AlexNet also uses the dropout layers to avoid the problem of overfitting.

\section{VGGNet}

As the second place of the ImageNet Challenge 2014, VGGNet [36] explores the relationship between the depth of convolutional neural networks and the performance of models. The depths of VGGNet range from 11 to 19 layers, the VGGNet16 and VGGNet19 are the common networks. The VGGNet16 is used in this study. The architecture of VGGNet16 consists of 13 convolutional layers, 5 max-pooling layers, 2 normalization layers, 3 fully connected layers and 1 softmax layer. The improvement of VGGNet is that consecutive $3 \times 3$ convolution kernels are used to replace the larger convolution kernels $(11 \times 11,7 \times 7,5 \times 5)$. The main purpose of consecutive $3 \times 3$ convolution kernels is to improve the effectiveness of the neural network by increasing the depth of the network under the condition of ensuring the same perceptual field. The VGGNet proves that the representation depth is beneficial to the classification accuracy.

\section{GoogLeNet}

Increasing network depth is an effective method to improve the performance of the deep neural network. However, with the increase of network layers, it will need a larger number of parameters and large computational resources. As the champion of 
ILSVRC14, GoogLeNet [37] proposes the Inception structure which improves the utilization of the network computing resources. The Inception module is assembled in parallel by multiple convolutions and pooling operations. The GoogLeNet network consists of the Inception modules stacked upon each other. The detailed introduction of the Inception module is reported in [37]. In this study, we use a common GoogLeNet architecture that consists of multiple Inception modules, which assembled in parallel by $1 \times 1,3 \times 3,5 \times 5$ convolutional filters and $3 \times 3$ max pooling.

ResNet

The depth of the neural network is important for model performance. However, the degradation problem occurs when the deeper network starts converging. As one of the breakthroughs works in the field of computer vision in recent years, ResNet [38] proposes a deep residual learning framework using shortcut connections to solve the degradation problem. The shortcut connection is an identity mapping that enables information to flow across layers without attenuation caused by nonlinear transformations of multiple stacks [39]. Two types of residual building blocks are proposed for different depth networks to reduce the number of parameters. For deeper networks, it consists of $1 \times 1,3 \times 3,1 \times 1$ convolutions and a shortcut connection. For shallow networks, it consists of $3 \times 3,3 \times 3$ convolutions and a shortcut connection. The depths of ResNet range from 18, 34, 50, 101 to 152 layers. The ResNet18 and ResNet50 are used in our study to recognize the rapeseed freezing injury.

\section{Experiment and Analysis}

In this study, we propose a method for freezing-tolerant rapeseed material recognition using UAV images and deep learning technology. In this section, to verify the effectiveness of our proposed method, we conduct the following experiments. Firstly, to prove the validity of the method and illustrate the performance of different networks, we train the five CNN models and test the performance of different models in section of Comparison of different CNNs. Secondly, to prove the performance of CNN models is better than the traditional machine learning, we compare the performance of four traditional machine learning models and the five CNN models in section of Comparison of traditional machine learning and CNNs. Thirdly, to test the effectiveness of the data augmentation strategy for CNN models, we compare the performance of the five models before and after data augmentation strategy in section of Illustration of data augmentation strategies. Finally, we use the new test site image collected by an UAV to test the generalization ability of different models in section of Experiment on the new test site. All CNN model architectures are implemented in Python using the PyTorch package version 1.19.0 on Ubuntu 16.04 operating system. The memory of the processor is $128 \mathrm{G}$, and the GPU is NVIDIA GTX 1080Ti. All traditional machine learning models are implemented in Python on Windows 10 operating system. It has a GPU of NVIDIA force RTX 3070, an Intel@ Core $^{\mathrm{TM}}$ i7-10700 CPU @ $3.20 \mathrm{GHz}$ with 128 GB RAM. Next, we will give a detailed introduction to these experiments. 


\section{Evaluation metrics}

The task of this study is to distinguish between freezing-injured materials and freezing-tolerant materials from a large number of candidates. The freezing injury recognition problem is a binary classification problem. To evaluate the performance of models used in our study, we use the common binary classification evaluation metrics, including accuracy, precision, recall and F-score. The four evaluation metrics are defined as:

$$
\begin{aligned}
& \text { accuracy }=\frac{T P+T N}{T P+T N+F P+F N} \\
& \text { precision }=\frac{T P}{T P+F P} \\
& \text { recall }=\frac{T P}{T P+F N} \\
& F-\text { score }=2 \times \frac{\text { precidion } \times \text { recall }}{\text { precidion }+ \text { recall }}
\end{aligned}
$$

where TP and TN denote the number of materials that is correctly predicted as freezing-tolerant materials and freezing-injured materials, respectively. FN denotes the number of materials that is incorrectly predicted as freezing-injured materials. FP denotes that the number of freezing-injured materials is incorrectly predicted as freezing-tolerant materials. The accuracy is the most intuitive evaluation metric for the overall performance of the model. However, it may fail when the number of samples from different categories is unbalanced. Thus, we further use the recall, precision and F-score to compare the performance of models. The precision is a metric that can indicate how many freezing-tolerant materials predictions are correct. The recall is the number of freezing-tolerant materials correctly predicted from all freezing-tolerant materials of the dataset. F-score is the harmonic mean of precision and recall.

\section{Comparison of different CNNs}

In this section, five common CNN models (AlexNet, VGGNet16, ResNet18, ResNet50 and GoogLeNet) are used to classify the freezing-injured materials and freezing-tolerant materials of rapeseed. In all experiments, we use the SGD optimizer with a learning rate of 0.01 , and the batch size of each model is 16 . Table 2 shows the comparison results of classification performance of different CNN models. Firstly, it can be seen that the accuracy of the five models is all over $92 \%$, which indicates that CNN algorithms show promise in the freezing injury recognition of rapeseed. Next, we can see that the ResNet50 achieves the highest accuracy (93.33\%) among the five models. However, the accuracy can be affected by unbalanced samples, it cannot fully reflect the performance of the CNN models. For example, in this study, the category we are interested in is freezing-tolerant materials. However, when the number of two categories is unbalanced that the number of freezing-tolerant materials is far less than freezing-injury materials, freezing-tolerant materials that are misclassified as freezing-injury materials can still make the model achieve high accuracy. Thus, we further use the recall, precision and F-score to compare the performance of CNN models. The model with a high recall (low FN) means that there is a large number of the freezing-tolerant materials recognized correctly by 
the model. The value of recall will be considered if the breeders want to find the maximum number of possible freezing-tolerant materials. The model with a high precision (low FP) means that there is a little number of freezing-injured materials recognized as freezing-tolerant materials. The breeders can quickly pick out the small amounts of freezing-injured materials from the result generated by deep networks. If we only consider the labor cost savings of selecting freezing-tolerant materials, ResNet18 will be recommended due to the highest precision (85.53\%). Because the aim of breeding is to select as many freezing-tolerant materials as possible. If only recall and precision are considered as evaluation metrics, recall is a more important evaluation metric for the recognition of the material. Thus, ResNet50 will be recommended because of the highest recall $(79.79 \%)$ while achieving high accuracy of $93.33 \%$. In the real situation, the breeders hope that they can quickly select more freezing-tolerant materials from candidates, the value of precision and recall should be all high. However, precision and recall are often conflicting. Hence, we need to balance two metrics. The F-score is a weighted average of precision and recall. If the difference between the values of $\mathrm{FP}$ and $\mathrm{FN}$ is large, then the $\mathrm{F}$-score should be considered firstly [40]. Among the five CNN models, ResNet50 obtains the highest F-score of $79.79 \%$. Overall, ResNet50 outperforms other CNN models for the freezing-tolerant rapeseed material recognition. From the above analysis, we can conclude that it is feasible for the freezing-tolerant rapeseed material recognition using UAV images and deep learning. In addition, the ResNet50 performs best among all selected networks. The ResNet50 offers the best scores of accuracy $(93.33 \%)$, recall $(79.79 \%)$ and F-score $(79.79 \%)$.

Table 2 Quantitative evaluation results of five CNNs.

\begin{tabular}{ccccc}
\hline Models & Accuracy(\%) & Recall $(\%)$ & Precision(\%) & F-score(\%) \\
\hline AlexNet & 92.63 & 73.40 & 80.23 & 76.67 \\
VGGNet16 & 93.16 & 73.40 & 83.13 & 77.97 \\
GoogLeNet & 92.45 & 67.02 & 84.00 & 74.56 \\
ResNet18 & 92.98 & 69.15 & $\mathbf{8 5 . 5 3}$ & 76.47 \\
ResNet50 & $\mathbf{9 3 . 3 3}$ & $\mathbf{7 9 . 7 9}$ & 79.79 & $\mathbf{7 9 . 7 9}$ \\
\hline
\end{tabular}

\section{Comparison of traditional machine learning and CNNs}

In this section, we compare the performance of four traditional machine learning models and five CNN models. In traditional machine learning models, the SIFT [41] and SURF [42] are selected as the hand-crafted features. SIFT and SURF are the two most widely used features and have been applied in many fields of agriculture, such as crop disease [40, 43], crop/weed classification [44], etc. In addition, the support vector machine (SVM) and artificial neural network (ANN) are selected as the classifier. The comparison results of four traditional machine learning models and five CNN models are shown in Table 3. As can be observed from the table, the evaluation metrics of the five CNN models are greater than those of the traditional machine learning models. The CNN networks significantly outperform the traditional machine learning methods in our task. Because the performance of traditional machine learning mainly relies on hand-crafted features, which are not optimal in almost all cases. Compared with traditional machine learning, deep learning does not need to manually design features. The features are automatically extracted using the CNN according to the task. Deep learning has a strong expression ability than traditional machine learning. 
Table 3 Comparison results between traditional machine learning and CNNs.

\begin{tabular}{cccccc}
\hline Type & Models & Accuracy(\%) & recall(\%) & precision(\%) & F-score(\%) \\
\hline \multirow{2}{*}{ traditional } & SIFT+SVM & 67.72 & 30.85 & 19.59 & $\mathbf{2 3 . 9 7}$ \\
machine learning & SURF+SVM & 61.05 & $\mathbf{3 6 . 1 7}$ & 17.35 & 23.45 \\
& SIFT+ANN & $\mathbf{7 6 . 3 2}$ & 15.96 & $\mathbf{2 1 . 4 3}$ & 18.29 \\
& SURF+ANN & 72.98 & 12.77 & 18.46 & 15.09 \\
\hline \multirow{4}{*}{ CNNs } & AlexNet & 92.63 & 73.40 & 80.23 & 76.67 \\
& VGGNet16 & 93.16 & 73.40 & 83.13 & 77.97 \\
& GoogLeNet & 92.45 & 67.02 & 84.00 & 74.56 \\
& ResNet18 & 92.98 & 69.15 & $\mathbf{8 5 . 5 3}$ & 76.47 \\
& ResNet50 & $\mathbf{9 3 . 3 3}$ & $\mathbf{7 9 . 7 9}$ & $\mathbf{7 9 . 7 9}$ & $\mathbf{7 9 . 7 9}$ \\
\hline
\end{tabular}

Illustration of data augmentation strategies

In this section, we compare the performance of five CNN models before and after data augmentation. We augment the dataset (2847 images) following the data augmentation strategy described in section of Dataset augmentation. After performing the data augmentation strategy, the number of the final dataset is expanded by 10 times, including 28470 images in total. The comparison results of the five CNN models before and after data augmentation are shown in Table 4. From the table, we can see that the accuracy of the five models is improved using the data augmentation strategy. However, the maximum improvement of the model accuracy is $0.53 \%$, which is relatively small. In addition, the other three evaluation metrics (recall, precision and F-score) do not improve significantly after using the data augmentation, and some of the models even show a decrease in the scores of evaluation metrics. It is shown that the data augmentation methods mentioned above have little effect on the accuracy of the freezing-tolerant rapeseed material recognition. The reason is that the boundary of the division between rapeseed freezing-injured and freezing-tolerant samples is not clear. There are some fuzzy data that cannot be accurately divided by experts. The increase of fuzzy data will affect the performance of the CNN models. We also observe that the ResNet50 outperforms the other CNN models after the data augmentation. It is consistent with the observation presented in section .

Table 4 Comparison results before and after data augmentation of five CNNs.

\begin{tabular}{cccccc}
\hline Dataset & Models & Accuracy(\%) & recall(\%) & precision(\%) & F-score(\%) \\
\hline \multirow{2}{*}{ original } & AlexNet & 92.63 & 73.40 & 80.23 & 76.67 \\
dataset & VGGNet16 & 93.16 & 73.40 & 83.13 & 77.97 \\
& GoogLeNet & 92.45 & 67.02 & 84.00 & 74.56 \\
& ResNet18 & 92.98 & 69.15 & $\mathbf{8 5 . 5 3}$ & 76.47 \\
& ResNet50 & $\mathbf{9 3 . 3 3}$ & $\mathbf{7 9 . 7 9}$ & 79.79 & $\mathbf{7 9 . 7 9}$ \\
\hline \multirow{3}{*}{ augmented } & AlexNet & 92.98 & 69.15 & 85.53 & 76.47 \\
dataset & VGGNet16 & 93.68 & 76.60 & 83.72 & 80.00 \\
& GoogLeNet & 92.80 & 71.28 & 82.72 & 76.57 \\
& ResNet18 & 93.33 & 70.21 & $\mathbf{8 6 . 8 4}$ & 77.65 \\
& ResNet50 & $\mathbf{9 3 . 6 8}$ & $\mathbf{7 9 . 7 9}$ & 81.52 & $\mathbf{8 0 . 6 5}$ \\
\hline
\end{tabular}

Experiment on the new test site

In this section, we use the images collected from a new site to test the generalization ability of the trained models. Generalization ability refers to the ability of a trained model that can make accurate predictions for the new data. We select 5 plots that contain 85 materials including 40 freezing-tolerant materials and 45 freezing-injured materials from the new test site. In Fig. 7, we present several images selected from the training dataset and the images of the new test site. We can observe that there 
is a large difference in the leaf feature between the training and test images. The overview of the new test site is shown in Fig. 8(a). The green boxes represent the freezing-tolerant materials, the blue boxes represent the freezing-injured materials.

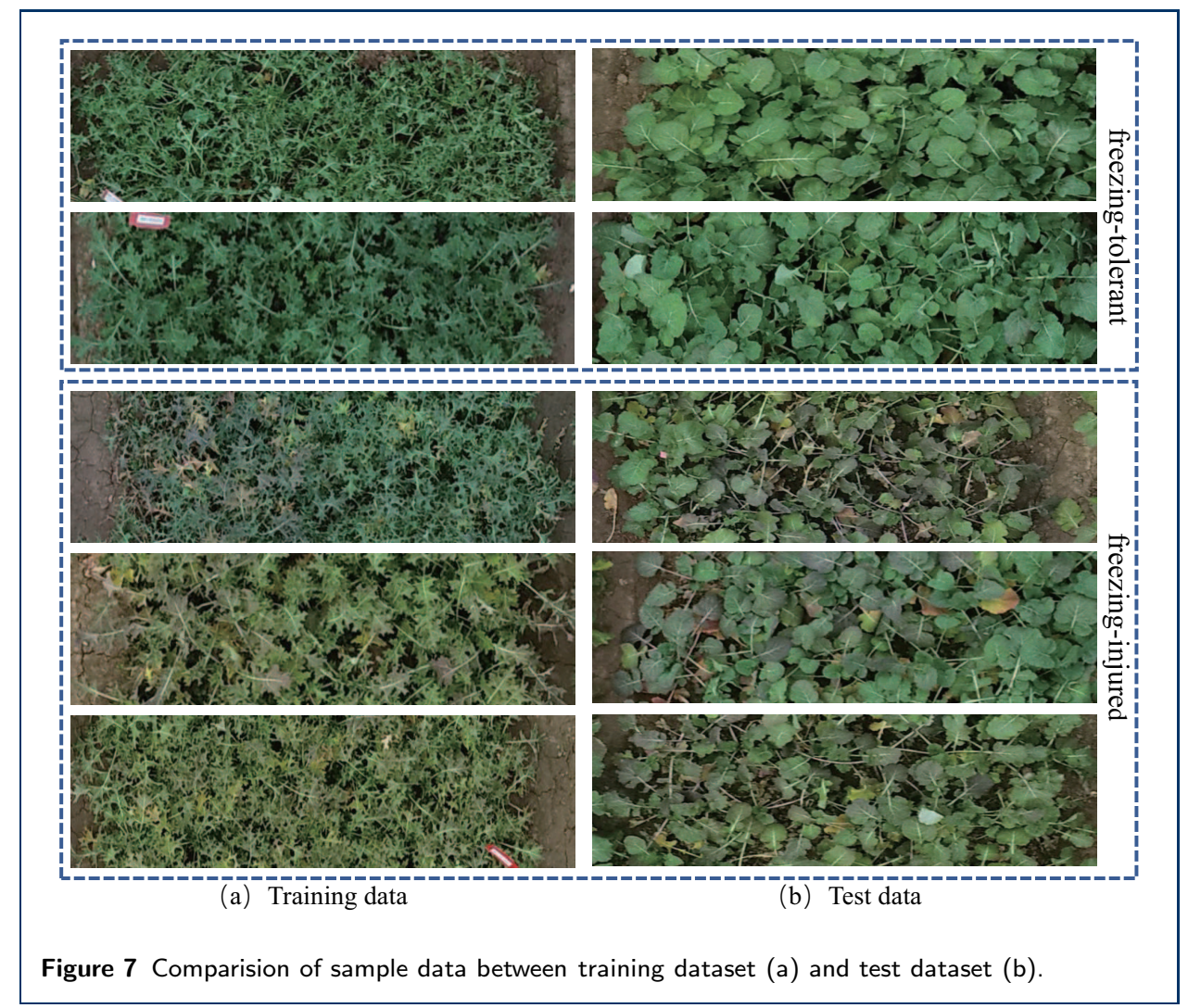

In the above experiments, the ResNet50 outperforms other CNN models, SIFT+ANN has the highest accuracy and SIFT+SVM has the highest F-score among the four traditional machine learning methods. Thus, we use the ResNet50, SIFT+ANN and SIFT+SVM to test the images collected from the new test site. The comparison results are shown in Table 5. Although there is a large difference in the training dataset and test dataset, the recognition performance of the CNN model outperforms traditional machine learning on the new dataset. The generalization ability of the ResNet50 can basically meet the requirement of freezing-tolerant rapeseed material recognition. Next, we will train the model using more overwintering rapeseed images collected in multiple regions and on multiple dates to further improve the recognition performance of the model.

The visualization result of ResNet50 is shown in Fig. 8(b). The red boxes and white boxes represent materials that are recognized incorrectly by the ResNet50, the number of each class is 7. By visualizing the experimental result of the model, we can visually observe that the freezing injury of rapeseed in large fields. Breeders can quickly assess and select freezing-tolerant materials based on the visualization result. In addition, breeders can take remedial action for early freezing-injured materials and reduce the loss of rapeseed freezing injury. The visualization of the rapeseed field can help managers for accurate management in complex and large-scale fields. 


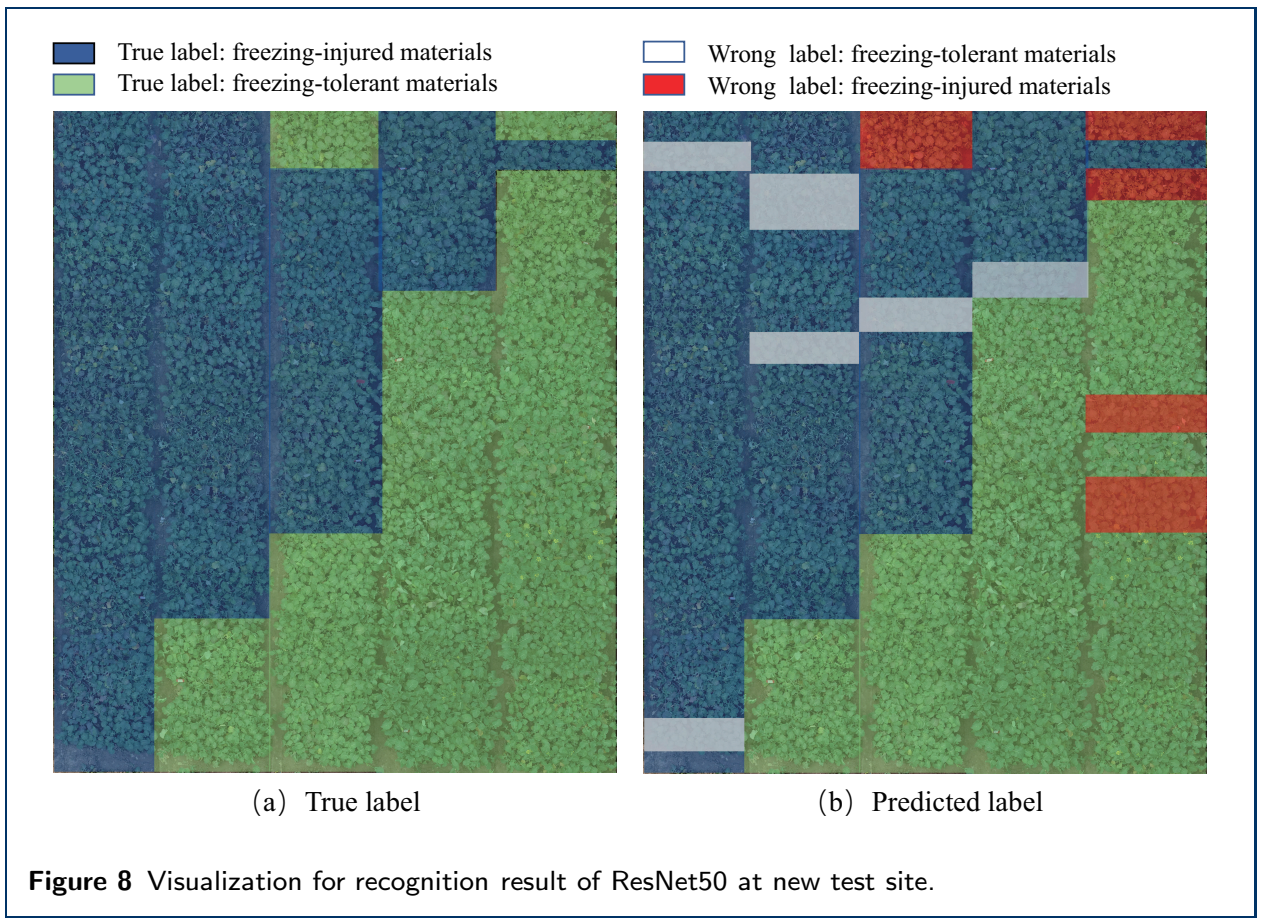

Table 5 Experimental results of three models at the new test site. (T-FT and T-FI denote that the true labels of rapeseed materials are freezing-tolerant and freezing-injured, respectively. P-FT and $\mathrm{P}-\mathrm{Fl}$ denote that the predicted labels of rapeseed materials are freezing-tolerant and freezing-injured, respectively.)

\begin{tabular}{cccccccc}
\hline Models & \multicolumn{1}{c}{ T-FT } & T-FI & Accuracy(\%) & Recall(\%) & Precision(\%) & F-score(\%) \\
\hline \multirow{2}{*}{ ResNet50 } & P-FT & 33 & 7 & $\mathbf{8 3 . 5 3}$ & $\mathbf{8 2 . 5 0}$ & $\mathbf{8 2 . 5 0}$ & \multirow{2}{*}{$\mathbf{8 2 . 5 0}$} \\
& P-FI & 7 & 38 & & & & \\
\hline \multirow{2}{*}{ SIFT+SVM } & P-FT & 23 & 15 & 62.35 & 57.50 & 60.53 & \multirow{2}{*}{58.97} \\
& P-FI & 17 & 30 & & & \\
\hline \multirow{2}{*}{ SIFT+ANN } & P-FT & 5 & 6 & 51.76 & 12.50 & \multirow{2}{*}{45.45} & \multirow{2}{*}{19.61} \\
& P-FI & 35 & 39 & & & & \\
\hline
\end{tabular}

\section{Conclusion}

This study combines deep learning technology and UAV images to build an AIassisted freezing-tolerant rapeseed material recognition model. This method can recognize freezing-tolerant materials from a large number of materials and further assist breeders to select breeding materials. In the study, the accuracy of the five CNN models is all over $92 \%$. In addition, ResNet50 achieves high accuracy of $93.33 \%$, while achieving the highest F-score of $79.79 \%$ and the highest recall of $79.79 \%$ among the other models. It proves that the feasibility of the freezing-tolerant rapeseed material recognition using UAV images combined with deep learning.

The freezing-tolerant rapeseed material recognition method established in this study has the advantages of economy, convenience, automation and high precision. Breeders not only can quickly assess and select freezing-tolerant materials but also can take remedial action for early freezing-injured materials to reduce the losses. This method can provide strong support for the scientific research work of rapeseed breeding and the study of the mechanism of freezing tolerance in rapeseed. Visualization of recognition result will further help breeders to achieve accurate management of rapeseed freezing injury. 
In this study, some fine results have been obtained, there are still some improvements that may be taken into consideration in the next work. In the future, we will collect rapeseed images during the overwintering period on multiple dates and in multiple regions to increase the diversity of data. In addition, we will divide the materials into more refined categories according to the freezing resistance of the materials, to further help the breeders for selecting the required materials more efficiently. We will also apply this method to other adversity monitoring and evaluation of rapeseed to promote accurate and intelligent development in the rapeseed field.

Acknowledgements

This work was partially supported by the Agricultural Science and Technology Innovation Program of the Chinese Academy of Agricultural Sciences (CAAS-ASTIP-OCRI), the National Natural Science Foundation of China (Project No. 31600179) and the Shenzhen Central Guiding the Local Science and Technology Development Program (No. 20211Szvup100)

Funding

Not applicable

Ethics approval and consent to participate

Not applicable...

Competing interests

The authors declare that they have no competing interests.

Consent for publication

Not applicable.

Author details

${ }^{1}$ School of Remote Sensing and Information Engineering, Wuhan University, Wuhan, China. ${ }^{2}$ GongQing Institute Of Science And Technology, Jiujiang, Jiangxi, China. ${ }^{3}$ Key Laboratory of Biology and Genetic Improvement of Oil Crops, Ministry of Agriculture and Rural Affairs, Oil Crops Research Institute of the Chinese Academy of Agricultural Sciences, Wuhan, China. ${ }^{4}$ School of Electrical and Electronic Engineering, Hubei University of Technology, Wuhan, China.

References

1. Fiebelkorn, D., Rahman, M.: Development of a protocol for frost-tolerance evaluation in rapeseed/canola (brassica napus I.). The Crop Journal 4(2), 147-152 (2016)

2. AghaKouchak, A., Farahmand, A., Melton, F., Teixeira, J., Anderson, M., Wardlow, B.D., Hain, C.: Remote sensing of drought: Progress, challenges and opportunities. Reviews of Geophysics 53(2), $452-480$ (2015)

3. Yoon, D.-H., Nam, W.-H., Lee, H.-J., Hong, E.-M., Feng, S., Wardlow, B.D., Tadesse, T., Svoboda, M.D., Hayes, M.J., Kim, D.-E.: Agricultural drought assessment in east asia using satellite-based indices. Remote Sensing 12(3), 444 (2020)

4. Vishnu, C., Sajinkumar, K., Oommen, T., Coffman, R., Thrivikramji, K., Rani, V., Keerthy, S.: Satellite-based assessment of the august 2018 flood in parts of kerala, india. Geomatics, Natural Hazards and Risk 10(1), 758-767 (2019)

5. Rahman, M.S., Di, L., Yu, E., Lin, L., Yu, Z.: Remote sensing based rapid assessment of flood crop damage using novel disaster vegetation damage index (dvdi). International Journal of Disaster Risk Science 12(1), 90-110 (2021)

6. Zhou, J., Pavek, M.J., Shelton, S.C., Holden, Z.J., Sankaran, S.: Aerial multispectral imaging for crop hail damage assessment in potato. Computers and Electronics in Agriculture 127, 406-412 (2016)

7. Bell, J.R., Gebremichael, E., Molthan, A.L., Schultz, L.A., Meyer, F.J., Hain, C.R., Shrestha, S., Payne, K.C.: Complementing optical remote sensing with synthetic aperture radar observations of hail damage swaths to agricultural crops in the central united states. Journal of Applied Meteorology and Climatology 59(4), 665-685 (2020)

8. Meichen, F., Chao, W., Wude, Y., Meijun, Z., Lujie, X.: Progress of remote sensing monitoring crop chilling and freeze injury. Journal of Shanxi Agricultural University (Natural Science Edition) (2014)

9. Allevato, E., Saulino, L., Cesarano, G., Chirico, G.B., D'Urso, G., Bolognesi, S.F., Rita, A., Rossi, S., Saracino, A., Bonanomi, G.: Canopy damage by spring frost in european beech along the apennines: effect of latitude, altitude and aspect. Remote Sensing of Environment 225, 431-440 (2019)

10. Feng, M.-C., Yang, W.-D., Cao, L.-L., Ding, G.-W.: Monitoring winter wheat freeze injury using multi-temporal modis data. Agricultural Sciences in China 8(9), 1053-1062 (2009)

11. She, B., Huang, J.-f., Guo, R.-f., Wang, H.-b., Wang, J.: Assessing winter oilseed rape freeze injury based on chinese hj remote sensing data. Journal of Zhejiang University-SCIENCE B 16(2), 131-144 (2015)

12. Wang, H., Huo, Z., Zhou, G., Wu, L., Feng, H.: Monitoring and forecasting winter wheat freeze injury and yield from multi-temporal remotely sensed data. Intelligent Automation \& Soft Computing 22(2), 255-260 (2016)

13. Cogato, A., Meggio, F., Collins, C., Marinello, F.: Medium-resolution multispectral data from sentinel-2 to assess the damage and the recovery time of late frost on vineyards. Remote Sensing 12(11), 1896 (2020) 
14. Perry, E.M., Nuttall, J.G., Wallace, A.J., Fitzgerald, G.J.: In-field methods for rapid detection of frost damage in australian dryland wheat during the reproductive and grain-filling phase. Crop and Pasture Science 68(6), 516-526 (2017)

15. Choudhury, B.U., Webster, R., Sharma, V., Goswami, J., Meetei, T.T., Krishnappa, R., Raju, P.L.: Frost damage to maize in northeast india: assessment and estimated loss of yield by hyperspectral proximal remote sensing. Journal of Applied Remote Sensing 13(4), 044527 (2019)

16. Goswami, J., Sharma, V., Chaudhury, B.U., Raju, P.: Rapid identification of abiotic stress (frost) in in-filed maize crop using uav remote sensing. International Archives of the Photogrammetry, Remote Sensing \& Spatial Information Sciences (2019)

17. Jełowicki, Ł., Sosnowicz, K., Ostrowski, W., Osińska-Skotak, K., Bakuła, K.: Evaluation of rapeseed winter crop damage using uav-based multispectral imagery. Remote Sensing 12(16), 2618 (2020)

18. Marin, D.B., Schwerz, F., Barata, R.A.P., de Oliveira Faria, R., Dias, J.E.L., et al.: Unmanned aerial vehicle to evaluate frost damage in coffee plants. Precision Agriculture, 1-16 (2021)

19. Revathi, P., Revathi, R., Hemalatha, M.: Comparative study of knowledge in crop diseases using machine learning techniques. Inter-national Journal of Computer Science and Information Technologies 2(5), 2180-2182 (2011)

20. Li, Y., Wang, H., Dang, L.M., Sadeghi-Niaraki, A., Moon, H.: Crop pest recognition in natural scenes using convolutional neural networks. Computers and Electronics in Agriculture 169, 105174 (2020)

21. Pantazi, X.E., Moshou, D., Alexandridis, T., Whetton, R.L., Mouazen, A.M.: Wheat yield prediction using machine learning and advanced sensing techniques. Computers and Electronics in Agriculture 121, 57-65 (2016)

22. van Klompenburg, T., Kassahun, A., Catal, C.: Crop yield prediction using machine learning: A systematic literature review. Computers and Electronics in Agriculture 177, 105709 (2020)

23. Rajapaksa, S., Eramian, M., Duddu, H., Wang, M., Shirtliffe, S., Ryu, S., Josuttes, A., Zhang, T., Vail, S., Pozniak, C., et al.: Classification of crop lodging with gray level co-occurrence matrix. In: IEEE Winter Conference on Applications of Computer Vision (WACV) (2018)

24. Zhang, Z., Flores, P., Igathinathane, C., L Naik, D., Kiran, R., Ransom, J.K.: Wheat lodging detection from uas imagery using machine learning algorithms. Remote Sensing 12(11), 1838 (2020)

25. Singh, A., Ganapathysubramanian, B., Singh, A.K., Sarkar, S.: Machine learning for high-throughput stress phenotyping in plants. Trends in Plant Science 21(2), 110-124 (2016)

26. Vakilian, K.A.: Machine learning improves our knowledge about mirna functions towards plant abiotic stresses. Scientific Reports 10(1), 1-10 (2020)

27. Ramos-Giraldo, P., Reberg-Horton, C., Locke, A.M., Mirsky, S., Lobaton, E.: Drought stress detection using low-cost computer vision systems and machine learning techniques. IT Professional 22(3), 27-29 (2020)

28. Su, J., Coombes, M., Liu, C., Zhu, Y., Song, X., Fang, S., Guo, L., Chen, W.-H.: Machine learning-based crop drought mapping system by uav remote sensing rgb imagery. Unmanned Systems 8(01), 71-83 (2020)

29. Firouz, M.S., Farahmandi, A., Hosseinpour, S.: Early detection of freeze damage in navel orange fruit using nondestructive low intensity ultrasound coupled with machine learning. Food Analytical Methods, 1-10 (2021)

30. Dargan, S., Kumar, M., Ayyagari, M.R., Kumar, G.: A survey of deep learning and its applications: a new paradigm to machine learning. Archives of Computational Methods in Engineering 27(4), 1071-1092 (2020)

31. An, J., Li, W., Li, M., Cui, S., Yue, H.: Identification and classification of maize drought stress using deep convolutional neural network. Symmetry 11(2), 256 (2019)

32. Noon, S.K., Amjad, M., Qureshi, M.A., Mannan, A.: Use of deep learning techniques for identification of plant leaf stresses: A review. Sustainable Computing: Informatics and Systems, 100443 (2020)

33. Yang, W., Yang, C., Hao, Z., Xie, C., Li, M.: Diagnosis of plant cold damage based on hyperspectral imaging and convolutional neural network. IEEE Access 7, 118239-118248 (2019)

34. Anami, B.S., Malvade, N.N., Palaiah, S.: Deep learning approach for recognition and classification of yield affecting paddy crop stresses using field images. Artificial Intelligence in Agriculture 4, 12-20 (2020)

35. Krizhevsky, A., Sutskever, I., Hinton, G.E.: Imagenet classification with deep convolutional neural networks. Advances in Neural Information Processing Systems 25, 1097-1105 (2012)

36. Simonyan, K., Zisserman, A.: Very deep convolutional networks for large-scale image recognition. arXiv preprint arXiv:1409.1556 (2014)

37. Szegedy, C., Liu, W., Jia, Y., Sermanet, P., Reed, S., Anguelov, D., Erhan, D., Vanhoucke, V., Rabinovich, A.: Going deeper with convolutions. In: IEEE Conference on Computer Vision and Pattern Recognition (CVPR) (2015)

38. He, K., Zhang, X., Ren, S., Sun, J.: Deep residual learning for image recognition. In: IEEE Conference on Computer Vision and Pattern Recognition (CVPR) (2016)

39. Targ, S., Almeida, D., Lyman, K.: Resnet in resnet: Generalizing residual architectures. arXiv preprint arXiv:1603.08029 (2016)

40. Shin, J., Chang, Y.K., Heung, B., Nguyen-Quang, T., Price, G.W., Al-Mallahi, A.: A deep learning approach for rgb image-based powdery mildew disease detection on strawberry leaves. Computers and Electronics in Agriculture 183, 106042 (2021)

41. Lowe, D.G.: Distinctive image features from scale-invariant keypoints. International Journal of Computer Vision 60(2), 91-110 (2004)

42. Bay, H., Tuytelaars, T., Van Gool, L.: Surf: Speeded up robust features. In: European Conference on Computer Vision (ECCV) (2006)

43. Raikar, K., Gawade, S., Turkar, V.: Usability improvement with crop disease management as a service. In: International Conference on Recent Innovations in Signal Processing and Embedded Systems (RISE) (2017)

44. Suh, H.K., Hofstee, J.W., IJsselmuiden, J., van Henten, E.J.: Sugar beet and volunteer potato classification using bag-of-visual-words model, scale-invariant feature transform, or speeded up robust feature descriptors and crop row information. Biosystems Engineering 166, 210-226 (2018) 